\title{
Correction to: Pratylenchus brachyurus parasitism on soybean: effects on productivity, vegetative and nematological parameters and chemical properties
}

\author{
Carla Marcondes Castanheira • Heloísa Gabriel Falcão • \\ Elza Iouko Ida • Claudia Regina Dias-Arieira • \\ Beatriz Cervejeira Bolanho Barros
}

Published online: 6 July 2020

(C) Koninklijke Nederlandse Planteziektenkundige Vereniging 2020

\section{Correction to: European Journal of Plant Pathology https://10.1007/s10658-020-02011-y}

This erratum is published as Third author name should be read as Elza Iouko Ida instead of Elza IoukoIda. Original article has been updated thus.

The online version of the original article can be found at https://doi.org/10.1007/s10658-020-02011-y

C. M. Castanheira $(\bowtie)$

Programa de Pós Graduação em Ciências Agrárias, Universidade Estadual de Maringá - UEM, Campus de Umuarama, Estrada da Paca s/n, CEP: 87500-000 Bairro São Cristóvão, Umuarama, PR, Brazil

e-mail: carlamarcas@hotmail.com

H. G. Falcão · E. I. Ida

Departamento de Ciência e Tecnologia de Alimentos/ Centro de Ciências Agrárias, Universidade Estadual de Londrina - UEL, Rodovia Celso Garcia Cid - Pr445 Km 380 Cx. Postal 10.011, Londrina, PR CEP: 86057-970, Brazil

C. R. Dias-Arieira

Departamento de Ciências Agronômicas, Universidade Estadual de Maringá - UEM, Campus de Umuarama, Estrada da Paca s/n, CEP: 87500-000 bairro São Cristóvão, Umuarama, PR, Brazil

B. C. B. Barros

Departamento de Tecnologia, Universidade Estadual de MaringáUEM, Av. Ângelo Moreira da Fonseca, 1800, Bairro: Zona VII, Umuarama, PR CEP: 87506-370, Brazil 